\title{
Optimal Distributed Coding Schemes for Energy Efficiency in the Fading Relay Channel
}

\author{
Fanny Parzysz \\ École de Technologie Supérieure \\ Montreal, Canada \\ E-mail: fanny.parzysz@lacime.etsmtl.ca
}

\author{
Mai H. Vu \\ McGill University \\ Montreal, Canada \\ E-mail: mai.h.vu@mcgill.ca
}

\author{
François Gagnon \\ École de Technologie Supérieure \\ Montreal, Canada \\ E-mail: francois.gagnon@lacime.etsmtl.ca
}

\begin{abstract}
We propose three energy-optimal distributed schemes for the half-duplex relay channel with block fading to maintain a desired source rate. We consider both network energy consumption and consumption of the relay alone, assuming only local channel knowledge. Then, we combine both into a generalized distributed energy-efficient scheme. In these schemes, the source uses message splitting and allocates resources dynamically, such that direct transmission, decode-forward or partial decode-forward is performed at each block, depending on the channel quality. The optimal distributed power allocation is computed from the corresponding centralized scheme by using an estimate of the relay consumption. Compared to decodeforward with no message splitting as often used in distributed designs, the proposed schemes significantly decrease the peak power and provide up to $15 \%$ average energy gain for the network consumption and a minimum of $3.3 \mathrm{~dB}$ gain for the relay consumption.
\end{abstract}

Keywords - energy consumption; fading relay channel; distributed power allocation

\section{INTRODUCTION}

Relayed communications allow cooperation among nodes to increase the performance in terms of diversity, achievable rate region, outage probability. Relaying has also been shown to improve network energy efficiency, and both advanced coding schemes and power allocations have been widely considered in the literature [1-7].

However, existing coding schemes [1,2] are usually centralized and assume global channel knowledge at all nodes. They thus require a large amount of feedback that may overload the network, and have to overcome potentially outdated measurements due to long propagation delay, such that they can be impractical to implement.

As a response to practical constraints, distributed power allocations have been considered, where each node uses only local channel knowledge. Nevertheless, most distributed algorithms are designed from the network perspective, such as relaying decision [3,4] or relay selection [5-7], and are based on suboptimal coding, such as repetition code or simple maximum ratio combining. Therefore, the potential energy gain is mainly obtained from the network topology, rather than from the relaying strategy itself, hence ignoring the fact that advanced coding can also bring significant gain over simple schemes as we have shown in [8] for the centralized case.

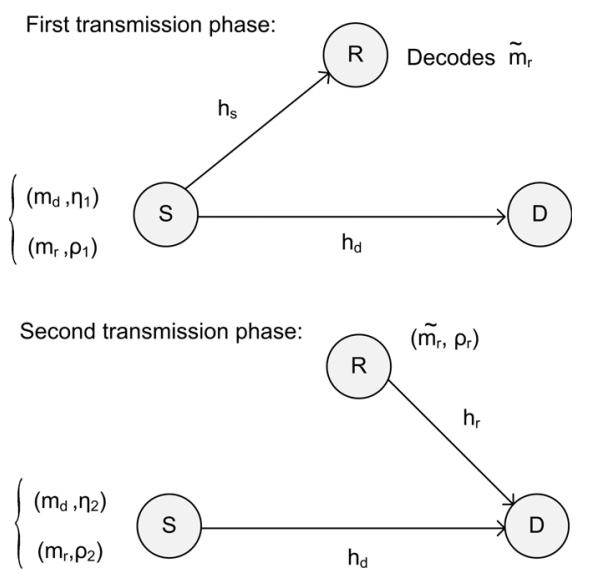

Fig. 1. A Half-Duplex Coding Scheme for Relay Channels

In this paper, we investigate coding and power allocation for the fading relay channel within the distributed constraint. Assuming only local channel knowledge, we identify the optimal coding strategy and distributed power allocations that minimize either the total network energy consumption or the relay consumption alone, while maintaining a desired source rate. For the network consumption, we first derive the distributed energy-optimal strategy which is based on the centralized scheme [8] and uses an estimate of the relay consumption. For the relay consumption alone, we show that the distributed scheme does not even require any link estimation. We next combine the proposed schemes into a generalized one that maximizes the energy-efficiency assuming only local channel knowledge. Last, we analyze the performance of the proposed distributed schemes as a function of the desired source rate.

\section{System Model for Fading Relay Channels}

\section{A. Channel model}

We consider a relay channel consisting of a source, a relay, and a destination. Let $h_{d}, h_{s}$ and $h_{r}$ respectively denote the flat fading coefficients of the direct link, the source-to-relay link and the relay-to-destination link, as depicted in Figure 1. These channel gains are zero-mean complex Gaussians with variances $\sigma_{d}^{2}, \sigma_{s}^{2}$ and $\sigma_{r}^{2}$, respectively. We consider independent AWGN $Z_{1}, Z_{2}$ and $Z_{r}$ each with variance $N=1$. 


$$
\begin{aligned}
& R \leq \theta \log _{2}\left(1+\frac{\left(\eta_{1}+\rho_{1}\right) P_{s}\left|h_{d}\right|^{2}}{N}\right)+\bar{\theta} \log _{2}\left(1+\frac{\left(\eta_{2}+\rho_{2}\right) P_{s}\left|h_{d}\right|^{2}+\rho_{r} P_{r}\left|h_{r}\right|^{2}+2 \sqrt{P_{s}\left|h_{d}\right|^{2} P_{r}\left|h_{r}\right|^{2} \rho_{2} \rho_{r}}}{N}\right)=I_{1}\left(\rho_{r}\left|h_{r}\right|^{2}\right) \\
& R \leq \theta \log _{2}\left(1+\frac{\rho_{1} P_{s}\left|h_{s}\right|^{2}}{N+\eta_{1} P_{s}\left|h_{s}\right|^{2}}\right)+\theta \log _{2}\left(1+\frac{\eta_{1} P_{s}\left|h_{d}\right|^{2}}{N}\right)+\bar{\theta} \log _{2}\left(1+\frac{\eta_{2} P_{s}\left|h_{d}\right|^{2}}{N}\right)=I_{2}
\end{aligned}
$$

\section{B. Coding scheme for the fading relay channel}

We consider the half-duplex partial decode-forward coding scheme presented in previous work [8] but propose new power allocations for the distributed constraint.

In this coding scheme, re-stated below for the benefit of the reader, the source and the relay have individual power constraints $P_{s}$ and $P_{r}$ within the same bandwidth. The transmission is half-duplex and is carried out in two phases within each code block of normalized length. During the first phase, of duration $\theta \in[0,1]$, the source transmits while the relay listens. During the second phase, of duration $\bar{\theta}=(1-\theta)$, both the source and the relay transmit. Each receiver can track the channel variations. We assume block fading such that the channel is constant over the two phases.

To send a message $m$ of rate $R$ to the destination, the source uses message splitting and divides the initial message into two parts $\left(m_{d}, m_{r}\right)$, with rates $R_{d}$ and $R_{r}$ respectively, where $R_{d}+R_{r}=R$. The message $m_{d}$ is directly decoded by the destination at the end of the second phase, whereas $m_{r}$ is intended to be relayed.

At each phase, each node allocates to each message a portion of its available transmit power. Denote $\eta_{1}$ and $\eta_{2}$ as the portions of source power $P_{s}$ allocated to $m_{d}$ in the first and second phase respectively; similarly, $\rho_{1}$ and $\rho_{2}$ as the portions for $m_{r}$. Denote $\rho_{r}$ as the portion of the relay power $P_{r}$ used to forward the decoded message $\tilde{m}_{r}$ to the destination. We consider transmit power constraint at each node such that

$$
\begin{aligned}
P_{s}^{(c)}=\theta\left(\eta_{1}+\rho_{1}\right) P_{s}+\bar{\theta}\left(\eta_{2}+\rho_{2}\right) P_{s} & \leq P_{s} \\
P_{r}^{(c)} & =\bar{\theta} \rho_{r} P_{r} \leq P_{r} .
\end{aligned}
$$

where the superscript ${ }^{(c)}$ refers to the consumed power. Denoting a codeword (frame) as $X^{n}=\left[X_{1}^{\theta n} X_{2}^{\bar{\theta} n}\right]$, the transmit signal in the two phases can be written as

$$
\begin{aligned}
& X_{r}=\sqrt{\rho_{r} P_{r}} U ; \quad X_{1}=\sqrt{\rho_{1} P_{s}} U+\sqrt{\eta_{1} P_{s}} V ; \\
& X_{2}=\sqrt{\rho_{2} P_{s}} U+\sqrt{\eta_{2} P_{s}} V
\end{aligned}
$$

where $U\left(m_{r}\right) \sim \mathcal{N}(0,1)$ and $V\left(m_{d}\right) \sim \mathcal{N}(0,1)$ are independent. The relay decodes $m_{r}$ at the end of the first phase and forwards it in the second phase. The destination, on the other hand, waits until the end of the second phase to jointly decode both $\left(m_{r}, m_{d}\right)$ based on the signals received during both phases.

All rates satisfying (3), at the top of the page, are achievable. Both rate constraints are results of the decoding at the relay over the first phase and joint decoding at the destination simultaneously over both phases. The first constraint captures the coherent transmission between the source and relay in the second phase, whereas the second constraint comes from the decoding of $m_{r}$ at the relay and decoding of $m_{d}$ at the destination.
In [8], we presented two energy-optimal centralized resource allocations that maintain a desired source rate and respectively minimize the total network energy consumption $(\mathrm{N}$ $\mathrm{EE})$ and the relay consumption alone (R-EE). These schemes require global channel information. Next, we discuss the distributed system model considered in this paper.

\section{System model for distributed strategies}

1) A model for channel knowledge: We consider local channel knowledge at all nodes, where each receiver has the channel state information (CSI) of the links to it, and each transmitter has CSI of the links from it. While the nextlink quality can be reasonably tracked through reciprocity or feedback over a reversed channel, a node may not be able to track the variations of distant links in time, such that this information cannot be used to compute power allocation. With this perspective, we assume that the source knows the gains $h_{d}$ and $h_{s}$ at each block, but it only knows the long-term statistics of $h_{r}$. Similarly, only the gains $h_{s}$ and $h_{r}$ are available at the relay. Furthermore, within the distributed constraint, each node performs power allocation separately. The source first computes estimates $\hat{\rho}_{r}\left|\hat{h}_{r}\right|^{2}$ of the relay consumption and unknown link, then allocates power based on its actual consumption and the relay expected consumption. The relay, however, can use the actual value of $h_{r}$ to allocate power.

2) Outage constraint: We propose two power allocations that respectively minimize the energy consumption of the whole network and of the relay alone, while maintaining a desired source rate and considering local channel knowledge. However, the distributed nature of the considered model impacts both the energy consumption and the outage probability. In both the distributed and centralized schemes, outage occurs at the source if the desired data rate is infeasible given the power constraints $P_{s}$ and $P_{r}$. However, the distributed scheme is subjected to a second outage event. Indeed, the local channel knowledge assumption implies a non-zero probability that the actual RD-link may not be strong enough to support the transmission of $m_{r}$ as expected by the source given its estimate $\hat{\rho}_{r}\left|\hat{h}_{r}\right|^{2}$, such that the outage probability of the centralized and distributed schemes cannot be equal considering the same power constraints. We finally assume that the source does not know at the end of phase 1 whether or not the relay will declare outage and keeps sending data during phase 2 anyway.

\section{DISTRIBUTED SCHEME FOR NETWORK ENERGY-EFFICIENCY DN-EE}

We first consider total source and relay power consumption during both transmission phases. Because of the distributed nature of the system, each node performs power allocation separately: the source allocates power to minimize the expected 
network consumption given the statistics of $h_{r}$, but the relay minimizes its consumption given the actual value of $h_{r}$.

\section{A. Problem setting for distributed power allocation}

Our goal is to find the optimal set $\left(\rho_{1}, \eta_{1}, \rho_{2}, \eta_{2}, \rho_{r}\right)$ that minimizes the total energy consumption with local channel knowledge, while maintaining a desired source rate $R$ :

$$
\begin{array}{ll}
\min & \mathrm{E}_{h_{r}}\left[P_{s}\left(\theta\left(\eta_{1}+\rho_{1}\right)+\bar{\theta}\left(\eta_{2}+\rho_{2}\right)\right)+P_{r} \bar{\theta} \rho_{r}\right] \\
& R \leq I_{1}\left(\rho_{r}\left|h_{r}\right|^{2}\right) \quad ; \quad R \leq I_{2} \\
\text { s.t. } & P_{s}^{(c)} \leq P_{s} \quad ; \quad P_{r}^{(c)} \leq P_{r}
\end{array}
$$

The two rate constraints ensure the achievability of the source rate, given the two individual power constraints. Note that the objective function can be decomposed into two separate parts: the source consumption, which depends on the statistics of $h_{r}$, and the relay consumption, which depends on the actual $h_{r}$. Next, we optimize each of these parts separately.

1) Optimization problem at the relay: The relay aims at minimizing its consumption based on the actual RD-channel gain, as well the source power allocation and message splitting determined at the beginning of phase 1 .

Proposition 1. Given the actual value for $h_{r}$, the optimal relay power allocation is such that the relay sends $\tilde{m}_{r}$ with power $\rho_{r} P_{r}=\hat{\rho}_{r} P_{r} \frac{\left|\hat{h}_{r}\right|^{2}}{\left|h_{r}\right|^{2}}$, where $\hat{h}_{r}$ is the source estimate of $h_{r}$ and $\hat{\rho}_{r} P_{r}$ is the source estimate of the relay power consumption.

Proof: The source rate $\mathrm{R}$ is achievable if $R \leq I_{1}\left(\left|h_{r}\right|^{2}\right)$. Note that the optimal resource allocation performed by the source given the estimate $\hat{h}_{r}$ leads to $R=I_{1}\left(\left|\hat{h}_{r}\right|^{2}\right)$. Therefore, to minimize its actual power consumption, the relay must set $\rho_{r}$ such that $I_{1}\left(\left|h_{r}\right|^{2}\right)=I_{1}\left(\left|\hat{h}_{r}\right|^{2}\right)$, leading to $\rho_{r}\left|h_{r}\right|^{2}=\hat{\rho}_{r}\left|\hat{h}_{r}\right|^{2}$.

Note that $\hat{\rho}_{r}\left|\hat{h}_{r}\right|^{2}$ is linked with the amount of information to relay. We assume here that $\hat{\rho}_{r}\left|\hat{h}_{r}\right|^{2}$ is forwarded by the source to the relay through a side channel or is encoded with the data (which only incurs a small overhead). Such techniques have been proposed in $[5,6]$ and keep the computation complexity at the source. Also note that the proposed distributed scheme does not require the relay to know the explicit value or statistics of $h_{d}$. This is beneficial, for example, in cellular networks where a fixed relay station serves many mobile users.

2) Relay expected consumption: As explained in Section II-C2, the actual RD-link may not be strong enough to support the transmission of $m_{r}$ as expected by the source given its estimate $\hat{\rho}_{r}\left|\hat{h}_{r}\right|^{2}$. The relay declares outage if its real consumption $\rho_{r} P_{r}$ does not satisfy the power constraint in (1), or equivalently if $\left|h_{r}\right|^{2}<\bar{\theta} \hat{\rho}_{r}\left|\hat{h}_{r}\right|^{2}$. Given the source estimate $\hat{\rho}_{r}\left|\hat{h}_{r}\right|^{2}$, the expected relay energy consumption can be written as follows:

$$
\begin{aligned}
\mathrm{E}_{h_{r}}\left[\bar{\theta} \rho_{r} P_{r}\right] & =\bar{\theta} \int_{\bar{\theta} \hat{\rho}_{r}\left|\hat{h}_{r}\right|^{2}}^{\infty} \rho_{r} P_{r} d\left|h_{r}\right|^{2} \\
& =\bar{\theta} \int_{\bar{\theta} \hat{\rho}_{r}\left|\hat{h}_{r}\right|^{2}}^{\infty} \hat{\rho}_{r} P_{r} \frac{\left|\hat{h}_{r}\right|^{2}}{\left|h_{r}\right|^{2}} \mathbb{P}\left(\left|h_{r}\right|^{2}\right) d\left|h_{r}\right|^{2} \\
& =\bar{\theta} P_{r} \frac{\hat{\rho}_{r}\left|\hat{h}_{r}\right|^{2}}{\sigma_{r}^{2}} \mathrm{E}_{1}\left(\bar{\theta} \frac{\hat{\rho}_{r}\left|\hat{h}_{r}\right|^{2}}{\sigma_{r}^{2}}\right)
\end{aligned}
$$

where $\left|h_{r}\right|^{2}$ follows an exponential distribution $\mathbb{P}\left(\left|h_{r}\right|^{2}\right)$ with parameter $\sigma_{r}^{2}$ and $\mathrm{E}_{1}$ refers to the exponential integral.

3) Optimization problem at the source: The source aims at finding the power allocation and relay estimate $\hat{\rho}_{r} \hat{h}_{r}$ such that the expected consumption of the network, i.e. sum of the source actual consumption and the relay expected consumption, is minimized:

$\begin{array}{ll}\min & P_{s}\left(\theta\left(\eta_{1}+\rho_{1}\right)+\bar{\theta}\left(\eta_{2}+\rho_{2}\right)\right)+P_{r} \frac{\bar{\theta} \hat{\rho}_{r}\left|\hat{h}_{r}\right|^{2}}{\sigma_{r}^{2}} \mathrm{E}_{1}\left(\bar{\theta} \frac{\hat{\rho}_{r}\left|\hat{h}_{r}\right|^{2}}{\sigma_{r}^{2}}\right) \\ \text { s.t. } & R \leq I_{1}\left(\hat{\rho}_{r}\left|\hat{h}_{r}\right|^{2}\right) ; \quad R \leq I_{2} ; \quad P_{s}^{(c)} \leq P_{s}\end{array}$

\section{B. Optimal distributed set of source power allocation}

Based on the above problem setting, we can adapt the centralized scheme of [8] to the distributed case as follows. The optimal distributed scheme for network energy efficiency, DN-EE, is such that,

- If $\left|h_{s}\right|^{2} \geq\left|h_{d}\right|^{2}$ and $R \geq R^{(d n)}$, then the source uses partial decode-forward (sub-scheme $A^{(d n)}$ ):

- In Phase 1, the source sends $m_{r}$ with power $\rho_{1}^{\star} P_{s}$;

- In Phase 2, the source sends $\left(m_{r}, m_{d}\right)$ with power $\left(\rho_{2}^{\star} P_{s}, \eta_{2}^{\star} P_{s}\right)$ and the relay sends $\tilde{m}_{r}$ with power $\rho_{r}^{\star} P_{r}$ when not in outage

- If $\left|h_{s}\right|^{2} \geq\left|h_{d}\right|^{2}$ and $R \leq R^{(d n)}$, then the source uses decode-forward (sub-scheme $B^{(d n)}$ ):

- In Phase 1, the source sends $m$ with power $\rho_{1}^{\star} P_{s}$;

- In Phase 2, the source sends $m$ with power $\rho_{2}^{\star} P_{s}$ and the relay sends $\tilde{m}$ with power $\rho_{r}^{\star} P_{r}$ if not in outage.

- If $\left|h_{s}\right|^{2} \leq\left|h_{d}\right|^{2}$ and $R \leq \log _{2}\left(1+\frac{P_{s}\left|h_{d}\right|^{2}}{N}\right)$, then the source uses direct transmission.

- Otherwise, the source declares outage.

Here, $R^{(d n)}=\theta \log _{2}\left(1+\frac{\rho_{1}^{\star} P_{s}\left|h_{s}\right|^{2}}{N}\right), \rho_{r}^{\star}$ is given by Proposition 1 . The sets $\left(\rho_{1}^{\star}, \rho_{2}^{\star}, \eta_{1}^{\star}, \eta_{2}^{\star}\right)$ for sub-schemes $A^{(d n)}$ and $B^{(d n)}$ are respectively given by Propositions 2 and 3 below.

Proposition 2. For a given $\theta$, the optimal power allocation set for sub-scheme $A^{(d n)}$ is

$$
\begin{aligned}
& \eta_{1}^{\star}=0 ; \quad \hat{\rho}_{r}\left|\hat{h}_{r}\right|^{2}=\frac{N}{P_{r}} \frac{\left(\frac{2^{R / \bar{\theta}}}{\left(1+\frac{\rho_{1}^{\star} P_{s}\left|h_{d}\right|^{2}}{N}\right)^{\theta / \theta}}-\frac{2^{R / \bar{\theta}}}{\left(1+\frac{\rho_{1}^{\star} P_{s}\left|h_{s}\right|^{2}}{N}\right)^{\theta / \theta}}\right)}{\left(1+g_{2}\left(\rho_{1}^{\star}\right)\right)^{2}} \\
& \eta_{2}^{\star}=\left(\frac{2^{R / \bar{\theta}}}{\left(1+\frac{\rho_{1}^{\star} P_{s}\left|h_{s}\right|^{2}}{N}\right)^{\theta / \bar{\theta}}}-1\right) \frac{N}{P_{s}\left|h_{d}\right|^{2}} ; \quad \rho_{2}^{\star}=\frac{P_{r}\left|\hat{h}_{r}\right|^{2}}{P_{s}\left|h_{d}\right|^{2}}\left(g_{2}\left(\rho_{1}^{\star}\right)\right)^{2} \hat{\rho}_{r} \\
& \text { where } g_{2}\left(\rho_{1}^{\star}\right)=\frac{\left(\frac{\left|h_{s}\right|^{2}}{\left|h_{d}\right|^{2}} \frac{2^{R / \bar{\theta}}}{\left(1+\frac{\rho_{1}^{\star} P_{s}\left|h_{s}\right|^{2}}{N}\right)^{1 / \bar{\theta}}}-1\right)}{\left(1-\frac{2^{R / \bar{\theta}}}{\left(1+\frac{\rho_{1}^{\star} P_{s}\left|h_{d}\right|^{2}}{N}\right)^{1 / \theta}}\right)}
\end{aligned}
$$


$\rho_{1}^{\star}$ is found numerically by solving $g_{1}\left(\rho_{1}^{\star}\right)=0$, with

$$
\begin{gathered}
g_{1}(s)=g_{2}(s)\left(\frac{2^{R / \bar{\theta}}}{\left(1+\frac{s P_{s}\left|h_{d}\right|^{2}}{N}\right)^{1 / \bar{\theta}}}-1\right)-1 \\
+\frac{\left|h_{s}\right|^{2}}{\left|h_{d}\right|^{2}} \frac{2^{R / \bar{\theta}}}{\left(1+\frac{s P_{s}\left|h_{s}\right|^{2}}{N}\right)^{1 / \bar{\theta}}}
\end{gathered}
$$

Proof: The proof uses Lagrangian techniques and is similar to Appendix B of [8].

If $\eta_{i}^{\star}$ and $\rho_{i}^{\star}$ do not satisfy the power constraints, the desired source rate cannot be achieved. Moreover, $\eta_{2}^{\star}$ is positive if $R \geq R^{(d n)}$ as defined in Proposition 2. If $R \leq R^{(d n)}$, we apply sub-scheme $B^{(d n)}$ as discussed next.

Proposition 3. The optimal power allocation for sub-scheme $B^{(d n)}$ is such that:

$$
\begin{aligned}
& \eta_{1}^{\dagger}=\eta_{2}^{\dagger}=0 ; \quad \rho_{1}^{\dagger}=\left(2^{R / \theta}-1\right) \frac{N}{P_{s}\left|h_{s}\right|^{2}} \\
& \rho_{2}^{\dagger}=\frac{P_{r}\left|h_{d}\right|^{2}}{P_{s}} \hat{\rho}_{r}\left|\hat{h}_{r}\right|^{2}\left(\frac{\bar{\theta} g_{1}\left(\hat{\rho}_{r}\left|\hat{h}_{r}\right|^{2}\right)}{\sigma_{r}^{2}}\right)^{2}
\end{aligned}
$$

where

$$
g_{1}\left(\hat{\rho}_{r}\left|\hat{h}_{r}\right|^{2}\right)=\mathrm{E}_{1}\left(\bar{\theta} \frac{\hat{\rho}_{r}\left|\hat{h}_{r}\right|^{2}}{\sigma_{r}^{2}}\right)-\exp \left(-\bar{\theta} \frac{\hat{\rho}_{r}\left|\hat{h}_{r}\right|^{2}}{\sigma_{r}^{2}}\right)
$$

and $\hat{\rho}_{r}\left|\hat{h}_{r}\right|^{2}$ is found numerically by solving

$\hat{\rho}_{r}\left|\hat{h}_{r}\right|^{2} P_{r}\left(1+\frac{\bar{\theta} g_{1}\left(\hat{\rho}_{r}\left|\hat{h}_{r}\right|^{2}\right)\left|h_{d}\right|^{2}}{\sigma_{r}^{2}}\right)^{2}=\left(\frac{2^{R / \bar{\theta}}}{\left(1+\frac{\rho_{1}^{\dagger} P_{s}\left|h_{d}\right|^{2}}{N}\right)^{\theta / \bar{\theta}}}-1\right)$

Proof: The proof uses Lagrangian techniques and is similar to Appendix B of [8].

Note that the scheme DN-EE is composed of the union of sub-schemes $A^{(d n)}$ and $B^{(d n)}$. The source does not need to find the explicit estimate of the RD-link $\hat{h}_{r}$ and only computes the product $\hat{\rho}_{r}\left|\hat{h}_{r}\right|^{2}$, which is forwarded by the source to the relay through a side channel or is encoded with the data.

\section{A GENERAL DistRIBUTED SCHEME FOR ENERGY-EFFICIENCY DG-EE}

In this section, we first propose a distributed scheme for relay energy-efficiency DR-EE. Next, we combine both schemes DN-EE and DR-EE into a generalized one DG-EE.

\section{A. Distributed scheme for relay energy-efficiency DR-EE}

We minimize the relay power consumption at each instant and allow the source to consume up to $P_{s}$ during both phases of the transmission.

Proposition 4. The distributed scheme optimal for the relay energy consumption (DR-EE) uses the same power allocation as the corresponding centralized scheme R-EE in [8].

Proof: See Appendix A.

Intuitively, to minimize the relay consumption, the source tries to send as much data as possible by itself. When the relay is needed to perform the transmission, the power allocation is already such that the message to relay is minimized, whatever the RD-link quality may be. As the power allocation does not require any estimation of $h_{r}$ at the source, the centralized and distributed schemes optimal for the relay energy consumption have the same outage probability and lead to the same average relay energy consumption. However, in the distributed scheme DR-EE, the source consumes more energy on average. Indeed, the centralized scheme declares an outage if the RD-link does not support the transmission of $\tilde{m}_{r}$. Nevertheless, this knowledge is not available in the distributed scheme so that the source transmits data in both phases whether or not the RD-link will be outage for $\tilde{m}_{r}$.

\section{B. General distributed scheme for network energy-efficiency $D G-E E$}

Similarly to the centralized scheme of [8], at the expense of consuming more total energy, DR-EE allows the source to reach higher rates than the maximal rate achieved by scheme DN-EE. We thus propose to combine DN-EE and DR-EE to achieve higher source rates.

Proposition 5. Considering total power consumption as a function of the source rate, the distributed resource allocation that minimizes the network consumption (DN-EE) is extended beyond its maximal achievable rate by the distributed allocation that minimizes the relay consumption (DR-EE).

Proof: See Appendix B.

Therefore, the generalized distributed scheme (DG-EE) optimal for energy-efficiency in the half-duplex relay channel with local channel knowledge is such that

- If $R \leq R^{(d n)}$, then apply $B^{(d n)}$

- If $R^{(\overline{d n})} \leq R \leq R_{\max }^{(d n)}$, then apply $A^{(d n)}$

- If $R_{\max }^{(d n)} \leq R \leq R_{\max }$, then apply $A^{(d r)}$

- Otherwise, declare an outage

where $R^{(d n)}$ is given in Section III-B, $R_{\max }^{(d n)}$ is the maximal rate achieved by DN-EE and $R_{\max }$ is the maximal rate achieved by DR-EE.

\section{PERformance ANALYsis}

\section{A. Reference schemes}

As reference, we consider direct transmissions (DTx), twohop routing (RTx) and distributed decode-forward with no message splitting (D-DF) as considered in [5, 6]. In RTx, the destination decodes data using the signal received in phase 2 only. Similarly to the proposed schemes, we assume local channel knowledge for all references and the source is allowed to send information to the relay via a side channel or via encoding. We equalize the outage probabilities of all schemes for each source rate to the outage probability of the general centralized scheme G-EE (proposed in [8]). To do so, we numerically adjust power constraints. 


\section{B. Comparison with reference schemes}

We consider the pure decode-forward scheme proposed in [5]. The source transmits the whole message to the relay during the first phase, while both the relay and destination are listening. During the second phase, only the relay transmits. As the source does not transmit in the second phase, no beamforming gain can be obtained. Then, maximum ratio combining is performed at the destination. The information required by the relay to perform optimal power allocation is forwarded on a side channel.

First, the coding scheme in [5] is equivalent to our scheme with $\rho_{2}=\eta_{1}=\eta_{2}=0$, such that it is a sub-case of subscheme $B^{(d n)}$, with rate constraints

$$
\begin{aligned}
& R \leq \theta \log _{2}\left(1+\frac{\rho_{1} P_{s}\left|h_{d}\right|^{2}}{N}\right)+\bar{\theta} \log _{2}\left(1+\frac{\rho_{r} P_{r}\left|h_{r}\right|^{2}}{N}\right) \\
& R \leq \theta \log _{2}\left(1+\frac{\rho_{1} P_{s}\left|h_{s}\right|^{2}}{N}\right)
\end{aligned}
$$

Note that the rate constraints are written in terms of SNR in [5], such that $S N R_{\text {target }}=\frac{\rho_{1} P_{s}\left|h_{s}\right|^{2}}{N}=2^{R / \theta}-1$ and $S N R_{\text {target }}^{\prime}=S N R_{\text {target }}-\frac{\rho_{1} P_{s}\left|h_{d}\right|^{2}}{N}$.

Second, the authors of [5] do not consider explicit power constraints such as (1) in this paper. This implies that the relay can always forward the source message, whatever the source allocation. Therefore, the optimization problem of [5] (eq. (58),(59) and (60)) considered a relaxed case of our problem and the allocation proposed in [5] is sub-optimal considering relay power constraints.

Thus, the scheme and optimal power allocation proposed in this paper include the scheme in [5] for a single relay model as a special case. Consequently, our scheme outperforms (or is at least as good as) the distributed decode-forward scheme [5] for all rates and all channel conditions. Similar analysis can show that our scheme also outperforms (or is at least as good as) direct transmissions and two-hop routing.

\section{Simulation results}

1) Average and peak total energy consumptions: Figure 2 depicts the average power consumption in the network, as a function of the source rate and confirms that the proposed scheme DG-EE outperforms other schemes. DG-EE provides more than $15 \%$ of energy gain compared to pure decodeforward. The gain reaches $4 \mathrm{~dB}$ compared to both direct transmissions and two-hop routing, which are mostly used in current systems allowing relaying.

In Figure 3, we plot the peak power consumption, i.e. the maximum energy that is punctually consumed by the source, or the relay, or both. We observe that the peak energy consumption of both the source and the relay, as well as the maximum total energy consumed in the network, are significantly higher for D-DF than for DG-EE, especially at low rates.

2) Average relay energy consumption: Figure 4 depicts the average energy consumed by the relay as a function of the source rate. The proposed distributed scheme DR-EE significantly outperforms both decode-forward and two-hop

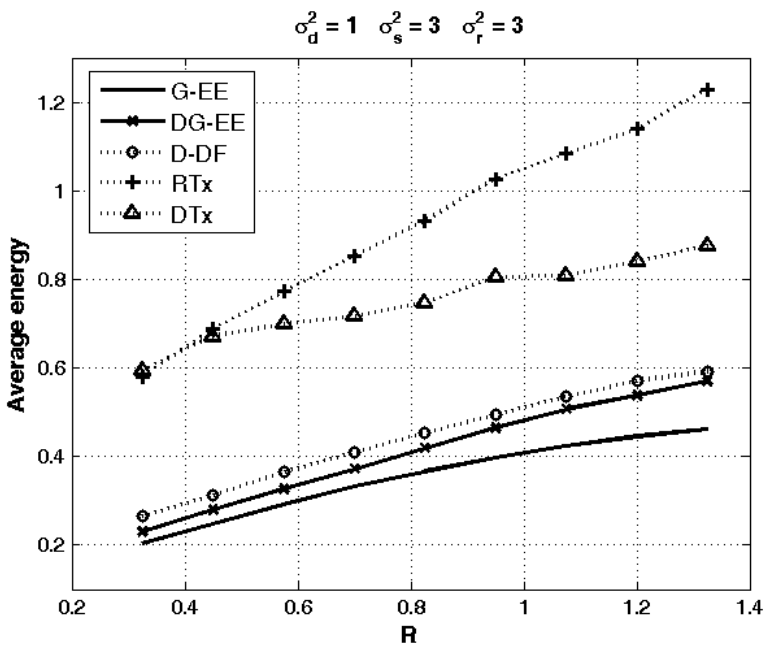

Fig. 2. Total energy consumption as a function of the source rate

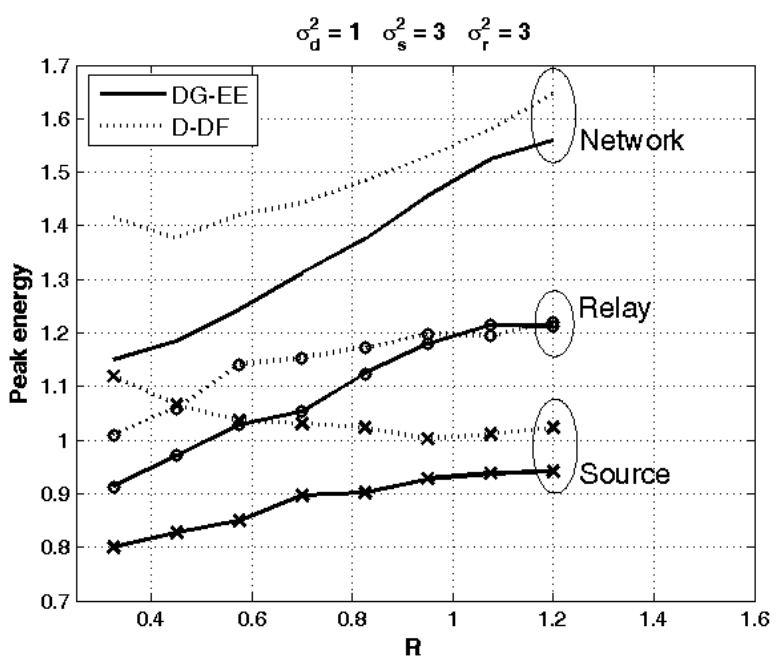

Fig. 3. Peak energy consumption as a function of the source rate

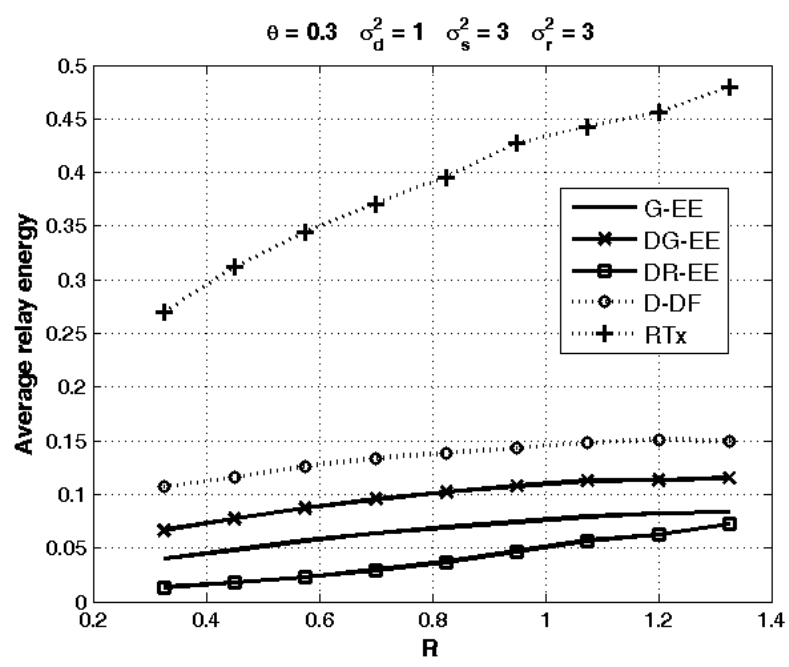

Fig. 4. Relay energy consumption as a function of the source rate 


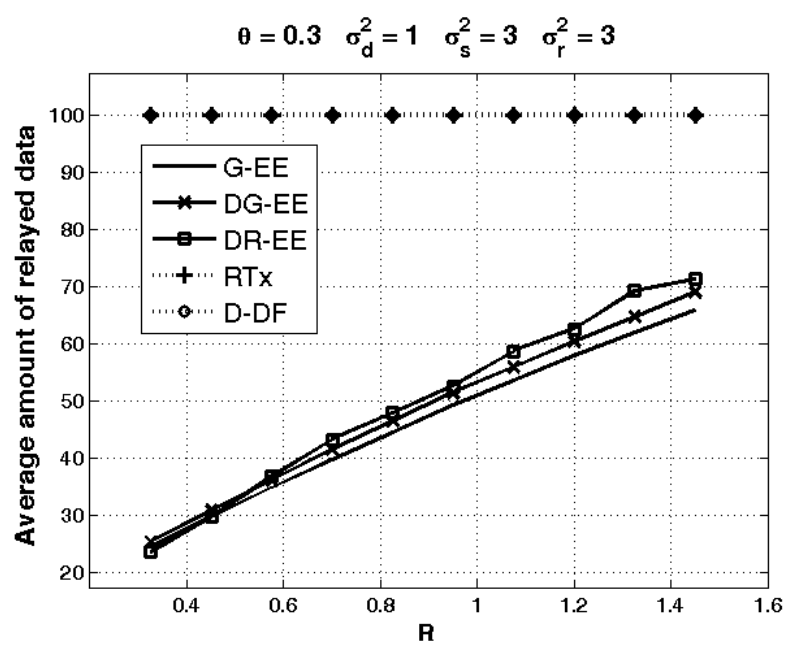

Fig. 5. Percentage of the source rate allocated to the relayed message

relaying. Compared to D-DF, the average relay energy gain is equal to $3.3 \mathrm{~dB}$ at high rates and reaches up to $6.5 \mathrm{~dB}$ at low rates, in which case the relay consumes almost no energy using the proposed scheme DR-EE.

3) Relay utilization: We compare the average amount of data that is relayed with the total amount of data that is transmitted per two-phase transmission block and plot in Figure 5 the average percentage of the source rate $R$ that is forwarded by the relay.

We can observe that the proposed distributed schemes relay on average a smaller amount of data compared to both decodeforward and two-hop routing, where the relay forwards the whole source message. This is particularly beneficial in a network with a shared relay station.

Moreover, in the distributed scheme optimized for relay consumption DR-EE, the relay forwards on average a larger amount of data compared to G-EE and DG-EE, which seems counter-intuitive since DR-EE is optimized for the relay energy. However, in the proposed schemes, the source and the relay coherently transmit $m_{r}$ during the second phase using powers $\rho_{2} P_{s}$ and $\rho_{r} P_{r}$ respectively. Then, the power allocation optimal for relay consumption shows that it is energy-efficient to increase the rate of $m_{r}$ but to allocate to it most of the source available power during the second phase. Hence, the ratio $\frac{\rho_{2} P_{s}}{\rho_{r} P_{r}}$ in DR-EE is significantly higher than in both G-EE and DG-EE.

\section{CONCLUSION}

In this paper, we explore the energy efficiency of partial decode-forward for the fading half-duplex relay channel within the distributed requirement. We propose two distributed schemes optimal for the network and the relay energy, which maintain a desired source rate and meet a target outage probability. We show that, compared to the coding schemes and power allocations used in existing distributed designs, both proposed schemes provide significant energy gain and decrease the peak power consumption of both the source and the relay, as well as the peak network energy consumption. As a result, this paper shows that advanced coding schemes can operate with local channel knowledge only and can significantly enhance the network performance.

\section{APPENDIX A}

\section{ProOF OF Proposition 4}

As developed in [8], the scheme R-EE is composed of three sub-schemes $A^{(r)}, B^{(r)}$ and $C^{(r)}$. We prove that each of these sub-schemes can be performed in a distributed manner.

First, $C^{(r)}$ corresponds to direct transmissions, where the relay is not used. Second, in $B^{(r)}$, note that the expressions of $\rho_{1}^{\dagger}$ and $\rho_{2}^{\dagger}$ do not depend on $h_{r}$. Since $\frac{\rho_{1}^{\dagger} P_{s}\left|h_{s}\right|^{2}}{N}$ is the received SNR, the relay can compute $\rho_{1}^{\dagger}$ and $\rho_{2}^{\dagger}$ as well and deduces the optimal $\rho_{r}^{\dagger}$. Finally, in sub-scheme $A^{(r)}$, note that

$\frac{P_{r}\left|h_{r}\right|^{2} \rho_{r}^{\star}}{P_{s}\left|h_{d}\right|^{2} \rho_{2}^{\star}}=\frac{N}{P_{s}\left|h_{d}\right|^{2} \rho_{2}^{\star}}\left(\frac{2^{R / \bar{\theta}}}{\left(1+\frac{\rho_{1}^{\star} P_{s}\left|h_{d}\right|^{2}}{N}\right)^{\frac{\theta}{\theta}}}-\frac{2^{R / \bar{\theta}}}{\left(1+\frac{\rho_{1}^{\star} P_{s}\left|h_{s}\right|^{2}}{N}\right)^{\frac{\theta}{\theta}}}\right)$

which does not depend on $h_{r}$. Therefore, the source can compute $\rho_{1}^{\star}, \rho_{2}^{\star}, \eta_{2}^{\star}$ and $\hat{\rho}_{r}^{\star}$ in a distributed manner. Knowing $\hat{\rho}_{r}^{\star}$ from the source and $h_{r}$ from reciprocity or feedback, the relay then computes the effective $\rho_{r}$.

\section{APPENDIX B}

\section{ProOF OF PROPOSITION 5}

We show here the continuity of sub-schemes $A^{(d n)}$ and $A^{(d r)}$ at $R=R_{\text {max }}^{(d n)}$. First, note that $\rho_{1}, \eta_{1}$ and $\eta_{2}$ solves the same equations for both sub-schemes, since $g_{2}\left(\rho_{1}^{\star}\right)=$ $\sqrt{\frac{P_{s}\left|h_{d}\right|^{2} \rho_{2}^{\star}}{P_{r}\left|h_{r}\right|^{2} \rho_{r}^{\star}}}$. Similarly, we can show that $\hat{\rho}_{r}$ of $A^{(d n)}$ and $\rho_{r}$ of $A^{(d r)}$ also solve the same equation.

Finally, at $R=R_{\max }^{(d n)}$, the source power constraint is met, such that $\rho_{2}^{\star}=\frac{\left(1-\rho_{1}^{\star} \theta\right)}{\theta}-\eta_{2}^{\star}$. Therefore, the optimal power allocation set for DN-EE is also optimal for DR-EE at this particular rate and both schemes are continuous.

\section{REFERENCES}

[1] A. Host-Madsen and J. Zhang, "Capacity bounds and power allocation for wireless relay channels," IEEE Trans. on Info. Theory, vol. 51, no. 6, pp. 2020 -2040, June 2005.

[2] Y. Yao, X. Cai, and G. Giannakis, "On energy efficiency and optimum resource allocation of relay transmissions in the low-power regime," IEEE Trans. on Wireless Comm., vol. 4, no. 6, pp. 2917 - 2927, Nov. 2005.

[3] H. Ning, C. Xiang, Z. Xiaofeng, S. Ben, L. Yunzhou, and W. Jing, "Energy-efficient relaying strategy with network coding in two-way parallel channels," ISRN Comm. and Networking, 2011.

[4] Z. Dawy and H. Kamoun, "The general gaussian relay channel: Analysis and insights," in 5th Int'l ITG Conf. on Source and Channel Coding (SCC), vol. 181. Margret Schneider, 2004, p. 469.

[5] M. Chen, S. Serbetli, and A. Yener, "Distributed power allocation strategies for parallel relay networks," IEEE Trans. on Wireless Comm., vol. 7, no. 2, pp. $552-561$, February 2008.

[6] H. Hui, S. Zhu, and G. Li, "Distributed power allocation schemes for amplify-and-forward networks," in IEEE Conf. on Wireless Comm. and Networking (WCNC), April 2009.

[7] S. Mallick, K. Kandhway, M. Rashid, and V. Bhargava, "Power allocation for decode-and-forward cellular relay network with channel uncertainty," in IEEE Int'l Conf. on Comm. (ICC), June 2011.

[8] F. Parzysz, M. Vu, and F. Gagnon, "Energy Minimization for the Half-Duplex Relay Channel with Decode-Forward Relaying," submitted to IEEE Trans. on Comm., under revision. [Online]. Available: http://arxiv.org/abs/1108.2829 